\title{
Design of Soil Moisture Detection and Control System
}

\author{
Chengfang Ji \\ School of Mechanical and Electrical Engineering, Zhengzhou University of Industry Technology, Zhengzhou \\ 450000, China \\ jichengfang8897@163.com
}

Keywords: Soil moisture, detection system, minimum system.

\begin{abstract}
Aiming at the problem of soil moisture detection and contro, the soil detection system was used as the research object, starting from the perspective of soil moisture, through the study of the soil moisture value, and using the analysis software to establish the model of the detection system, which are carried out the simulation calculation. It can be proved that soil moisture can be tested and controlled, which has a reference value for future research on soil moisture.
\end{abstract}

\section{Introduction}

Humidity is the most basic unit for measuring soil moisture. A certain volume of water content in the air is a basic unit for measuring humidity. The less moisture in the air, the drier the air in the environment, also, the more water in the air, the more humid the air. With the progress of science and technology, people have developed a low-power, high-performance soil detection and control system. The system can monitor soil humidity in real time and solve the soil humidity monitoring problem for farmers, and realize automatic irrigation, which is convenient and efficient. Therefore, the research of humidity detection and control system has been paid more and more attention. This design also considers this reason, and further study and study of humidity control system.

The system realizes soil moisture detection, if the current soil moisture is less than the set of soil moisture is open alarm, alarm LED light is lit at the same time, this time of the relay, absorption by relay control simulation and water, at this time in soil and water inside, the soil humidity rising. When the soil humidity is higher than the set limit, disconnect the relay and shut off the buzzer.

\section{System Design Scheme}

Soil moisture detection is mainly divided into two parts: software design and simulation design. The final two parts are combined to complete soil moisture detection and control. The various functions of soil moisture detection and control are realized by debugging and testing. At the same time, LCD1602 display, key adjustment, alarm and LED instruction are implemented. The detection signal is entered into a single chip microcomputer, and the output unit is followed by a single chip microcomputer to achieve different control processes for different functions or units.

This paper is based on STC89C52, which used to control the humidity measurement and control system to achieve its performance target. The key to the system is the automatic control of soil moisture. In this aspect SCM has its own unique advantage, price is cheap, control aspect, easy to program, has better stability.

The minimum system includes power supply, reset circuit and concussion circuit. The MCU reset is to enable the CPU and other system features to return to a determined initial state in the system and run from the initial state to the startup, for example, when MCU resets PC0000H, the SCM system unit starts fetching instructions. The main control system circuit is shown in figure 1. 


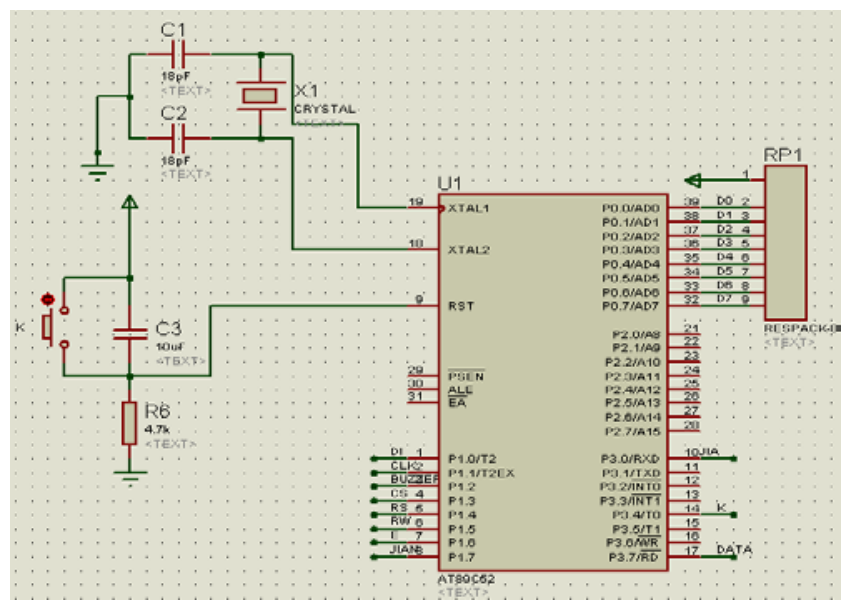

Fig. 1 The main control system circuit

\section{The method}

The control unit of soil moisture detection and control system is single chip microcomputer. The design adopts STC89C52 as the MCU unit, Software programming uses keil software for programming, Using Protues7.5 to carry out the system simulation test experiment. In terms of system programming, A/D conversion process is the key and difficult point for the design and programming unit, and key control is also important and difficult in the programming design, in order to convenient to observe and control system of humidity information information through LCD1602 display and set the upper and lower limits. Program logic sequence, an infinite loop in the main from top to bottom, when the interrupt or key changes, save the breakpoints, response has a priority order, call interrupt first, and then call button, when the interrupt and button continue to return after the breakpoint continue execution. When all programming debugging generates hex files after compilation, the hex file is manually added to the MCU via Protues7.5 software. In the simulation software, the humidity control system is tested.

\subsection{System Humidity Normal Simulation Stage}

The simulation of normal working of system humidity is shown in figure 2. RH_L displays the current buttons to adjust the value of, the $\mathrm{RH}$ is greater than the $\mathrm{RH} \_\mathrm{L}$, namely the measured soil moisture value is greater than the set value, can see the buzzer alarm light is closed, and simulate the LED water light is closed. Indicates that the soil moisture content is in normal state.

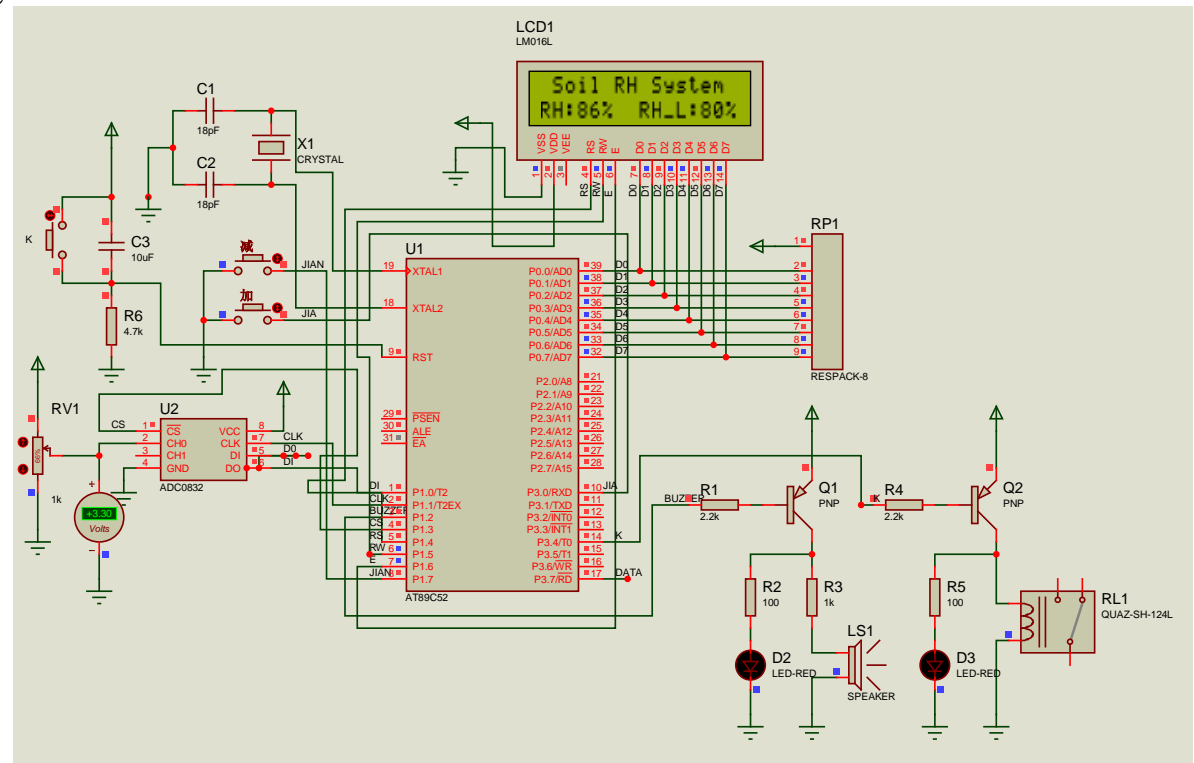

Fig. 2 System humidity normal working condition 


\subsection{System Alarm Simulation Stage}

Soil moisture detection control system with single chip as the CPU unit, the soil humidity sensor to collect the soil moisture, the collection of data sent to ADC0832 conversion chip, send the converted analog signals to single chip microcomputer, single chip microcomputer based on the analysis of data collected by the humidity. If RH is less than RH_L, the collected soil moisture is less than the current setting humidity, and the microcontroller sends the instructions to the buzzer, the buzzer alarm and the LED light. At the same time, the microcontroller sends the control dc to the relay, and the water is added to the soil by a relay absorption simulation.and the LED indicator light is lit. The current soil moisture and the setting of soil moisture are shown in LCD1602. In any process, you can use the key to add and subtract the humidity value. System simulation is shown in figure 3.

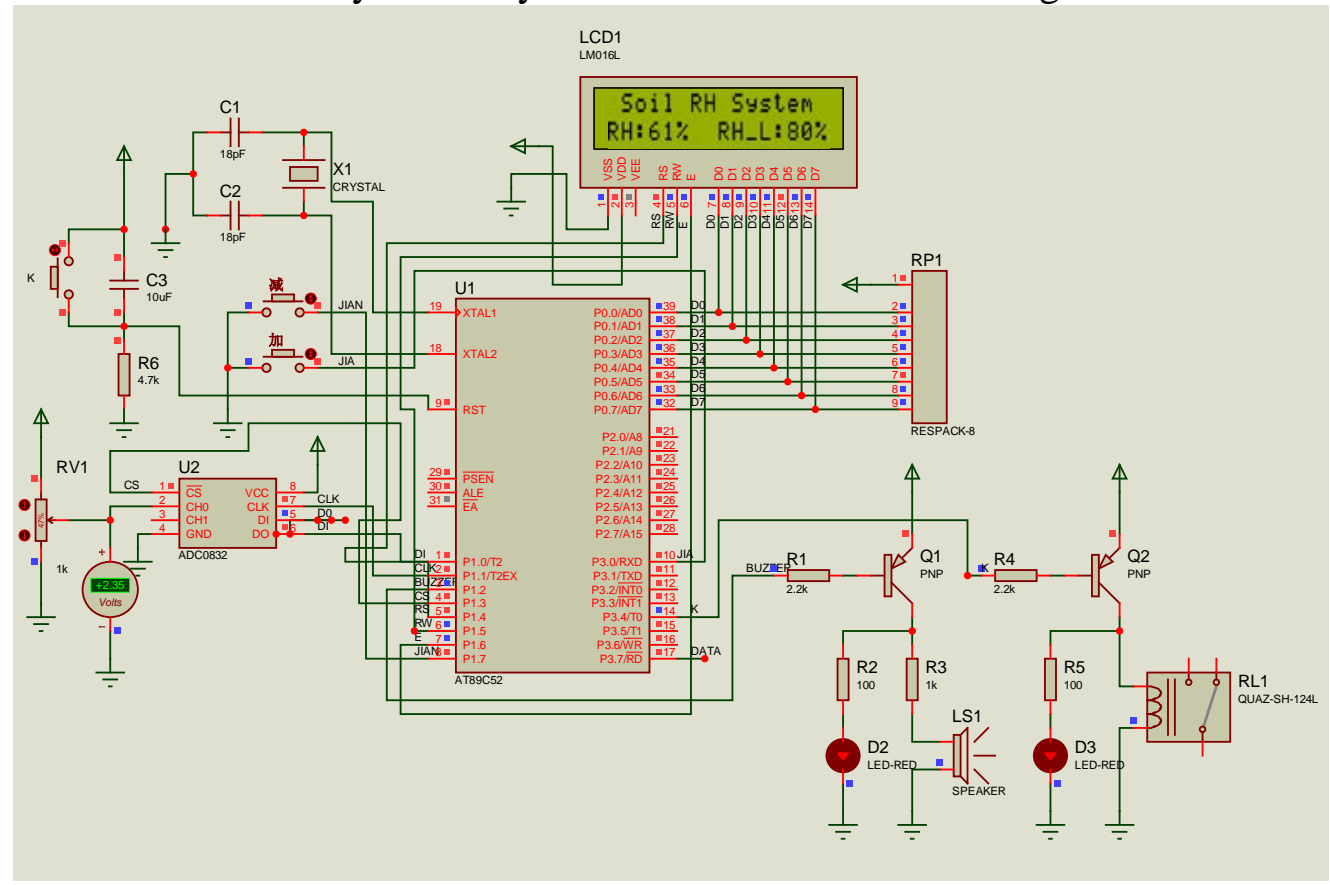

Fig. 3 System alarm simulation diagram

\section{Conclusions}

Through the analysis of normal phase and alarm stage of simulation system, the detection and control of soil moisture is realized. Different humidity can be set up to test the system, which has certain reference value for future research on soil moisture.

\section{References}

[1] Fang zipeng, Huang shuangping, Chen zhongtao, Design of soil humidity control system based on single chip planter[J]. Modern Agricultural Equipment,2013(4):41-45.

[2] Zhou Xiaobo, Li Ji, the design of soil moisture automatic control system The design of multi-channel remote irrigation control system based on greenhouse crops Journal of fuyang normal university,2012,29(2):24-28.

[3] Chen Shiguo, Hu Lihua, Wu Dongsheng, etc. Design of the following soil temperature control system[J]. Information and Communication,2015(11):77-79.

[4] Niu Tao, Wang Yiya, Chen Shuguang, etc. The design of multi-channel remote irrigation control system based on greenhouse crops[J]. Modern Agricultural Technology,2017(2):147-148.

[5] Tan Xiaogang, Gao Guowei, Zhang Liqiang, etc. The design of agricultural soil moisture controller based on Arduino platform[J]. Sensor World,2016 ,22(5):30-34. 